\title{
Germanica
}

GERMANICA $7 \mid 1990$

Grenze und Entgrenzung

\section{Ilse Tielschs Grenzüberschreitungen}

Carine Kleiber

\section{OpenEdition}

Journals

Édition électronique

URL : http://journals.openedition.org/germanica/2498

DOI : 10.4000/germanica.2498

ISSN : 2107-0784

\section{Éditeur}

Université de Lille

\section{Édition imprimée}

Date de publication : 30 juin 1990

Pagination : 87-96

ISSN : 0984-2632

\section{Référence électronique}

Carine Kleiber, « Ilse Tielschs Grenzüberschreitungen », Germanica [Online], 7 | 1990, Online erschienen am: 17 Juli 2014, abgerufen am 06 Oktober 2020. URL : http://journals.openedition.org/germanica/ 2498 ; DOI : https://doi.org/10.4000/germanica.2498

Ce document a été généré automatiquement le 6 octobre 2020.

(c) Tous droits réservés 


\title{
Ilse Tielschs Grenzüberschreitungen
}

\author{
Carine Kleiber
}

1 Ilse Tieisch, 1929 in Mähren geboren, betrat die Bühne des Literaturbetriebes erstmals im Jahre 1966, also relativ spät, als sich ihre künstlerischen Ambitionen endlich mit den Pflichten einer Ehefrau und Mutter mehrerer Kinder vereinbaren ließen. Sie veröffentlichte den Lyrikband In meinem Orangengarten sowie den Prosatext Brief ohne Anschrift. Darauf folgten bis 1975 die Gedichtsammlungen Herbst mein Segel (1967), Anrufung des Mondes (1970) und Regenzeit (1975). In der zweiten Hälfte der siebziger Jahre verlegte Ilse Tieisch den Schwerpunkt ihrer Tätigkeit auf die Kurzprosa, wobei sie sich zunehmend auf ihre Vergangenheit bezog. 1974 war Begegnung in einer steirischen Jausenstation erschienen. Es folgten 1975 Erinnerung an Großvater, 1977 die satirischen Erzählungen Ein Elefant in unserer Straße, und 1979 Zirkusgeschichte sowie das Kleinod Erinnerung mit Bäumen. Dieses letzte Werk bereitete das zeitgeschichtliche Tryptichon Die Ahnenpyramide (1980) Heimatsuchen (1982) und Die Früchte der Tränen (1988) vor. Ein kleines Intermezzo in dieser umfangreichen historischen Freske stellten der Lyrikband Nicht beweisbar und die ebenfalls 1981 erschienene Erzählung Die Königin mit den goldenen Haaren dar. Die Erzählung Fremder Strand (1984) erschloß einen neuen Themenkreis, indem er die Krise einer reifen Frau darstellt. Ihr folgten als bisher letzte Publikationen der Gedichtband Zwischenbericht (1986) und der Erzählband Der Solitär (1987) ${ }^{1}$.

Ein ins Haus gekommener Fragebogen ${ }^{2}$ und ein enttäuschendes Wiedersehen mit der Heimat, bei dem sie feststellen mußte, daß der Ort, dem ihre ganze Sehnsucht gegolten hatte, zu einem ihr fremden tschechischen Nest geworden war ${ }^{3}$, forderten Ilse Tieisch zu einer direkten Auseinandersetzung mit dem Heimatbegriff auf:

Heimat ist jener Ort, an dem mein Bewusstsein geprägt worden ist, sagt ein Schulfreund zu mir, als wir beisammensitzen und versuchen, uns an Damals zu erinnern, an die Dörfer und kleinen Städte, in denen wir als Kinder und junge Leute gelebt haben, die Landschaften, die sie umgaben, haben uns ihre Zeichen mitgegeben, wir sprechen ihre Sprache noch, wir erlernen die neuen Dialekte nicht, wo auch immer wir seither gelebt haben, wo wir heimisch geworden sind...

B. ist dem Kind Anni Heimat gewesen. [...] Ich weiß, daß ich durch alles, was ich dort erlebt habe, durch die ersten Jahre meines Lebens, durch meine Erinnerung an diese Jahre, gefüßhlsmäßig an B. und an das Umland von B. gebunden bin. Trotzdem gelingt es mir nicht, den Begriff Heimat für mich selbst ausschließlich auf 
diesen kleinen Bereich zu konzentrieren.

(Alte Heimat und neue Heimat, die Frage, ob Heimat ausschließlich gleichzusetzen sei mit dem Land der Herkunft, ob nicht auch jener Ort, an dem man seßhaft geworden ist, [...] Heimat genannt werden muß...) $)^{4}$.

Die Ahnenpyramide und Heimatsuchen setzen immer wieder zu neuen Versuchen einer Definition der Heimat an. Obwohl dieser Begriff für Ilse Tieisch gewiß weiterhin mit für mährische Landstriche typischen Maisfeldern, Hügellandschaften oder Winzerund Jagdtreiben verknüpft bleibt, orientiert sich ihr Heimatbegriff nicht mehr nur an der Vergangenheit und an dem Ort, der mit ihr zusammenhängt. Er wird ergänzungsfähig und bedürftig, insofern als er die Gegenwart miteinbezieht, und er erfährt, was sein vergangenheitsbezogenes Moment anbelangt, wegen der Diskrepanz zwischen Heimatvorstellung und heutiger Wirklichkeit, eine Verinnerlichung. Die sogenannte alte Heimat entzieht sich einer genauen örtlichen Festlegung und läßt private, sachlich anfechtbare Assoziationen wie jene böhmischen KriStalls mit Mähren zu.

Die intensive Beschäftigung mit dem Heimatbegriff und gefühl ist bei Ilse Tieisch von einer Konfrontation mit der Vergänglichkeitsproblematik begleitet. Ihre Angst vor dem gänzlichen Auslöschen, d.h. vor der totalen Vergeblichkeit irdischen Lebens kommt häufig zum Ausdruck ${ }^{5}$. Das Gedicht "Gewöhne dich nicht»" erweist sich jedoch als konstruktiv, indem es das Schreiben als eine sich der Vergessenheit widersetzenden Beschäftigung darstellt. Ilse Tieisch macht es sich zum Ziel, Erlebtes der Vergänglichkeit zu entreißen, indem sie es in eine dauerhafte Form bringt. Handelt es sich dabei um längst Vergangenes, so kommt das Schreiben nicht nur auf ein Benennen, sondern auf ein Heraufbeschwören heraus, mit dessen Problematik sie sich insbesondere in Erinnerung mit Bäumen, Die Ahnenpyramide und Heimatsuchen beschäftigt. Dabei ist ihr Augenmerk auch auf die kommenden Generationen gerichtet, denen sie einerseits das längst Geschehene näher bringen will, und in die sie sich andererseits auch durch ihre Zeugenschaft projiziert ${ }^{7}$. Diese Auffassung rührt von jener einer engen Generationsverkettung her, die Ilse Tieisch in Die Ahnenpyramide erörtert:

Wir können längere Zeit so leben, als gäbe es das Vergangene nicht, als wären nur Gegenwart und Zukunft wichtig, aber die Vergangenheit holt uns ein. [...] Plötzlich wissen wir, daß wir Glieder einer Kette sind, die weit in die Vergangenheit zurückreicht und sich im Zukünftigen fortsetzen wird... ${ }^{8}$.

Die Generationsverbundenkeit liegt vor allem in einer genetischen Übertragung physischer, charakterlicher, geistiger und künstlerischer Veranlagungen. Ilse Tieisch glaubt angesichts des beinahe zyklischen Charakters der menschlichen (Un)Taten nicht an einen Fortschritt der Menschheit. Aber sie verwirft die Idee einer Erbbarkeit von Verschulden'.

Der Umgang mit dem Gewesenen verleitet Ilse Tieisch weiter zu einer Untersuchung des menschlichen Erinnerungsvermögens und seiner Beeinflussung durch die Einbildungskraft, welche sie nicht nur bewußt als Belebungsmöglichkeit einer «toten», weil von ihr weder unmittelbar noch mittelbar erlebten, ja meist auf Grund der zeitlichen Entfernung erlebbaren Vergangenheit einsetzt, sondern von der sie, besonders im Falle von Sehnsucht, das Überrumpelungsvermögen kennt, um es am eigenen Leibe beim Wiedersehen mit der mährischen Heimat erfahren zu haben. Sie weiß um die tröstende, also oft lebenserhaltende Funktion der die Wirklichkeit beschönigenden Phantasie, die sich bei normal-vitalen Menschen z.B. als Ausgleich für einen das seelische Gleichgewicht bedrohenden Verlust einstellt ${ }^{10}$. Sie bezeichnet das entstandene Wunschbild sogar als korrigierte und korrigierende Wirklichkeit ${ }^{11}$ womit 
sie sich offenbar für die Fiktion und gegen die Realität ausspricht, eine Feststellung, die insofern mit dem Wahrheitsanspruch ihrer geschichtsthematischen Bücher $\mathrm{zu}$ vereinbaren ist, als die Realität für sie aus unbestreitbaren, weil durch aufwendiges Archivmaterial überprüften Tatsachen, zusammengesetzt ist.

7 Sie weiß nicht minder um die Unverläßlichkeit der Erinnerung selbst, die womöglich ebenfalls aus Gründen des menschlichen Erhaltungstriebes, oder zumindest des Dranges nach Unbeschwertheit in den meisten Fällen schmerzliche Erlebnisse verdrängt ${ }^{12}$, und stellt fest, daß Geräusche und Gerüche nicht nur Erinnerungen auslösen, sondern auch deren Gegenstand ausmachen können ${ }^{13}$. Sie veranschaulicht den raffenden und schematisierenden Charakter der Erinnerungen, die Daten und Ereignisse übereinanderschieben, die unterschiedlichsten sinnlichen Erfahrungen verbinden ${ }^{14}$, und deren Genauigkeit mit der Vergrößerung des zeitlichen Abstandes zunimmt ${ }^{15}$. Ihre Beobachtung, nach der sie sich nur noch selten in das Kind oder das junge Mädchen, das sie einmal war, hineindenken vermag, daß dessen Erlebnisse und Gedanken ihr fremd geworden $\operatorname{sind}^{16}$, läßt auf eine allmähliche objektivierung der Erinnerung im Sinne eines unbewußten, emotionalen Rückzugs aus ihnen schließen. Mit ihrer in Erinnerung mit Bäumen gestellten Frage nach den Kriterien für die (Nicht)Abrufbarkeit vergangener Erlebnisse ${ }^{17}$ versucht sie zumindest einen Denkanstoß zur weiteren gründlichen Untersuchung des Erinnerungsvermögens zu liefern.

Dort, wo keine Erinnerung vorhanden ist, weil sie nicht vorhanden sein kann oder will, und Ilse Tieisch auch keinen individuellen, d.h. anekdotischen und differenzierten Zeugenbericht betsitzt, greift sie auf die ihr eigenen Technik des Heraufbeschwörens zurück, die z.T. in einem tranceartigen Zustand, z.T. auch nur dank ihrer aus dokumentarisch abgesicherten Phantasie genährten Einfühlungsgabe erfolgt. In jedem Falle vertieft sie sich dann in eine Zeichnung, oder taucht in ein Photo ein. Mit Hilfe einer Lupe, deren Wirkung oft noch mit einem Augenglas verstärkt wird, überwindet sie den üblichen Abstand zwischen dem Betrachtenden und dem betrachteten Bild, wechselt von einem objektiven, überlegenen Blick auf einen Gegenstand zu einem subjektiven Rundblick auf eine unmittelbare Welt über. Dann wird das Bild plastisch, d.h. "dreidimensional» ${ }^{18}$, und die sich somit selbst in Szene setzende Autorin vollbringt gleichzeitig einen Sprung in die Vergangenheit, überlistet die Zeit. Der Zauber dauert Sekunden oder auch Minuten, bis er aus schriftstellerischer Notwendigkeit abgebrochen, oder auch nur unterbrochen wird:

ich muß mir ins Gedächtnis zurückrufen, warum ich hier stehe, mitten im zweiten Drittel des neunzehnten Jahrhunderts, in der Gegenwart derer, die vor mir gewesen sind

liest man in Die Ahnenpyramide ${ }^{19}$.

Dieser poetische Vorgang einer sich stets wiederholenden Bildgestaltung ergibt eine parataktische Fügung, die den Texten, ob Gedicht oder Prosa, einen eher statischen Charakter verleiht, der durch gleichlautende Einleitungsworte zu den nebeneinander gestellten Bildern oder Szenen, in der Prosa manchmal auch nur durch die regelmäßige Wiederholung eines Schlüsselgedankens, noch verstärkt wird. Die leitmotivische Evozierung eines oberösterreichischen Bauernhofes und der ihm zugehörigen Obstbäume in Erinnerung mit Bäumen ${ }^{20}$, der Die Ahnenpyramide durchziehende Blick auf ein Photo oder eine Zeichnung, z.B., erfüllen aber gleichzeitig eine einheitsbildende Fuktion in diesen wegen des Mangels an Spannungselementen vom Auseinanderfallen bedrohten Texten. Vom schreibtechnischen Standpunkt her, bedarf es dieses 
Kunstgriffes, um den willentlich unterbrochenen Redefluß nicht versanden zu lassen. Hier kann von der Dynamik der Wiederholungen gesprochen werden, die stets das für eine Neubeginn nötige Stichwort liefern. Auf Gedichte angewandt, ergeben parataktische Fügung und Wiederholung einen Rhythmus, einen Wohlklang, die Ilse Tieisch im Zyklus «Lirum larum Löffelstiel» aus dem Band Anrufung des Mondes als Verfremdungsode besser Befremdungseffekt verwendet. Die Tatsachen und Zukunftsvisionen, die sie in diesen musikalischen, an und für sich verspielten, an Abzählreime angelehnten Gedichten liefert, zählen zu den bittersten ${ }^{21}$.

11 Nicht nur bei der Suche nach ihrer Vergangenheit bezieht Ilse Tieisch ungewöhnliche Posten, rüttelt sie an der Zeitlichkeit. Die Anleitung einer Bronzestatue befolgend, projiziert sie sich in Vorlauf iger Auf enthalt (Zwischenbericht) aus der Froschperspektive von ihrem Arbeitszimmer in die Unendlichkeit. Im Watt bei Cuxhaven (Zwischenbericht) ist der Versuch, durch die Vermittlung einer vereinigten Reflektierung zweier verschiedener Raumebenen, die raumtiefe zu negieren:

die Augen im Spiegel

starren mich an

aus dem Abgrund des Himmels

und um die Spuren der Jahre an einem menschlichen Körper zu veranschaulichen, schrumpft die Autorin in Das Gesicht des Malers H.P. (Nicht beweisbar) zu einem Wesen zusammen, das einen Spaziergang durch die Furchen eines Gesichts zu unternehmen vermag.

Im Rahmen ihrer Auseinandesetzung mit dem Altern greift Ilse Tieisch in drei Fallen aus verschiedenen Schaffensperioden auf das Motiv des Welkens der Haut zurück, das sie im Zeitrafferverfahren veranschaulicht:

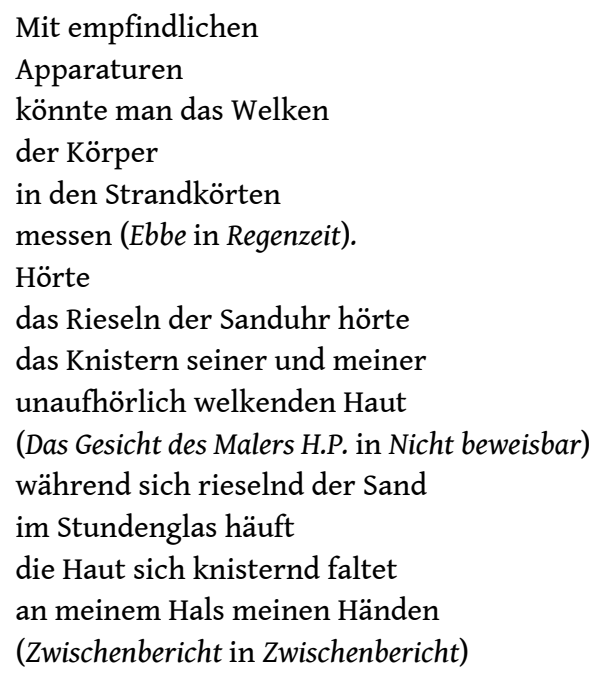

Die untermalte Geräuschkulisse als Ergebnis auditiver Phantasie-Eindrücke des Gedächtnisses unterstützt das Vorstellungsvermögen der Auswirkung einer zeitlichen Beschleunigung.

Insbesondere im jüngst erschienenen Band Zwischenbericht wählt die Autorin auffallend häufig einen entrückten Blickwinkel und greift somit auf eine Technik zurück, die jener des Eintauchens in eine bildlich oder gedanklich vorhandene Szene geradezu entgegengesetzt ist. So entsteht ein «kosmisches» Weltbild, das den Menschen und die Welt stets im Rahmen des Universums und im Verhältnis zu ihm zeigt ${ }^{22}$. Auch in rein existentiellen Gedichten, in denen sich das für Ilse Tielschs Schreibkunst so typische 
visuelle Moment nur als Vision einstellen kann, wird diese Perspektive gern beibehalten. Ein Beispiel hierfür aus einer weiter zurückliegenden Schaffenszeit liefert Enttäuschung morgen (Anrufung des Mondes):

Im Staub der Fixsterne

eine sichtbare

Fußspur: Godot

ist weitergegangen

Die bildliche Nachweisung ihrer meist von Trauer angesichts der Eitelkeit menschlichen Handelns, der Nichtigkeit der Welt und ihrer Bewohner sowie deren Gottesverlassenheit gezeichneten Intuitionen und Gedanken bedarf eines über das übliche Maß der dichterischen Sprachgestaltung hinausgehenden freien Umgangs mit allen Bereichen unseres Weltverständnisses. In Weinviertel nördlich oder in Auf der Deichkrone stehend bei Flut (beide in Zwischenbericht wird das Unsichtbare sichtbar gemacht:

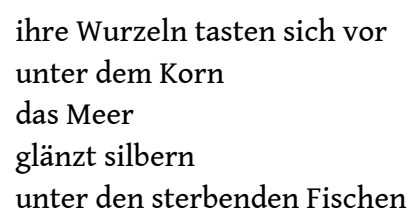

In Schlafstörung nachts (Zwischenbericht) vermenschlicht die Autorin Europa und meint das Stöhnen ihres von Kriegserinnerungen geplagten Kontinents zu hören.

Ilse Tielschs Spiel mit den Perspektiven und Größenordnungen, die Fäden, die sie von der Vergangenheit in die Gegenwart spinnt oder auch umgekehrt, die Art und Weise, wie sie das Begriffliche, ihre Vorstellungen ins Bildliche übersetzt - all dies beweist, daß sie die Geltungsbereiche, ja sogar die Berechtigung der Grundformen unserer Welterfahrung in frage stellt. Die Grenzüberschreitungen sind Aufbegehrungsversuche gegen die einengenden Gesetze unserer Existenz und unseres Daseins, die Ilse Tieisch in ihrer Diesseitigkeit sehr jung, und später in ihrem vollen Maße besonders schmerzlich (sie verlor zwei ihrer Kinder) zu erfahren hatte. Ilse Tieisch experimentiert die Entgrenzung, weil sie ihr Leben lang an Grenzen gestoßen ist.

\section{NOTES}

1. - «In meinem Orangengarten», Bergland Verlag, Wien 1964. - «Brief ohne Anschrift», Marburger Bogendrucke, 1966. - «Anrufung des Mondes», Jugend \& Volk, Wien 1970. «Regenzeit», Styria Verlag, Graz 1975. «Begegnung in einer steirischen Jausenstation», Neugebauer-Press, Bad Goisern, 1974 - «rinnerung an Großvater», Marburger Bogendrucke, 1975. - «Ein Elefant in unserer Straße», Styria Verlag, Graz 1977. - «Zirkusgeschichte», Marburger Bogendrucke, 1979. - «Erinnerung mit Bäumen», Styria Verlag, Graz 1979. - «Die Ahnenpyramide», Styria Verlag, Graz 1980. - «Nicht beweisbar», Delp Verlag, München 1981. «Heimatsuchen», Styria Verlag, Graz 1982. - «Die Königin mit den goldenen Haaren», Marburger Bogendruck, 1983. «Fremder Strand», Styria Verlag, Graz 1984. - «Zwischenbericht», Grasl, Baden bei Wien 1986. - «Der Solitär», Styria Verlag, Graz 1987. - «Die Früchte der Tränen» Styria 
Verlag, Graz 1988.

Die in Klammern vermerkten Seitenzahlen beziehen sich auf diese Ausgaben.

2. - Vgl. «Die Ahnenpyramide» S. $28 \mathrm{f}$.

3. - Ebenda S. 430-432.

4. - «Die Ahnenpyramide» S. 235, 289 - «Heimatsuchen» S. 149.

5. - Vgl. z.B. das Gedicht «Vita mit Nachsatz» in «Nicht beweisbar»: «Geboren ein Namen genannt fremd geblieben verloren gegangen Der Schatten dort auf dem Weg das könnte ich sein vielleicht».

6. - In «Anrufung des Mondes».

7. - In einem Brief an die Verfasserin dieses Textes schreibt Ilse Tieisch:

Was ich schreibe, ist nicht von Sehnsucht erfülltes Zurückdenken, sondern genaue und engagierte Spurensuche. [...] Die Vergangenheit, die mich betrifft (nicht nur mich natürlich) wird bewußt aus den Geschichtsbüchern, von den Landeskarten, aus dem Bewußtsein der Menschen, gelöscht. Weil ich darüber Bescheid weiß, versuche ich, so so viel mir nur möglich ist, davon aufzuspüren und in meinen Texten festzuhalten. Ich tue dies nicht mit Sehnsucht, sondern mit Akribie. [...] Ich halte den Schritt, der historisch vollzogen wurde, für endgültig und glaube, daß man ihn akzeptieren muß. Was erarbeitet werden müßte, wäre ein neues Verständnis derer für einander, die nach jahrhundertelangem Miteinanderleben (und Gegeneinanderleben im gleichen Land) durch die historischen und politischen Ereignisse getrennt worden sind. Ich schreibe also für mich, um mich selbst zu finden, aber auch für meine Kinder, um ihnen zu sagen, woher sie kommen und wer sie sind. Daß ich damit nicht nur meine leiblichen Kinder meine, ist hoffentlich klar.

8. - «Die Ahnenpyramide» S. 115.

9. - Vgl. «Die Ahnenpyramide» S. 201: «Ich bin bereit, sie zu akzeptieren, die vor mir gewesen sind, als jene, ohne die ich nicht wäre, ich nehme sie an, ich versuche nicht, ihre Existenz zu leugnen, ich trenne mich nicht von ihnen Sorgen, ich trage keine Verantwortung für ihre Schuld...».

10. - Vgl. «Heimatsuchen» S. 184.

11. - «Heimatsuchen» $S, 143$.

12. - Vgl. z.B. «Erinnerung mit Bäumen» S. 103: «Dieses sicherlich furchtbare Erschrecken ist jedoch aus meiner Erinnerung ausgespart gewesen und auch der Schmerz, den ich damals mit Sicherheit empfunden habe» oder «Die Ahnenpyramide» S. 149: «Heinrich, der Vater, hat die Schattenstellen aus den Bildern seiner Erinnerung längst getilgt. Vielleicht kehren die Ängste, die Schrecken, die Verzweiflungen [...] in den Nächten wieder...». Ein Gegenbeispiel befindet sich in «Heimatsuchen» S. 20: «Der Schmerz ist abgeklungen, Schrecken und Angst jedoch blieben unvergessen».

13. - Vgl. z.B. «Erinnerung mit Bäumen», S. 75: «Diese Bilder, sagte Anna, seien von Geräuschen, von Stimmen, ja sogar von Gerüchen begleitet gewesen», oder «Die Ahnenpyramide» S 40: «Ich sage Leinen und weiß mehr als andere, die ebenfalls Leinen sagen. Ich höre, rieche, taste dieses Wort, ich sehe Farben, die mit dem Wort in Verbindung stehen, auf deen Hintergrund das Wort schwimmt...», oder «Heimatsuchen» S. 418: «Die Erinnerung an den Geschmack ist geblieben. Der Kaffee hat wie Zichorie geschmeckt, sagt die Mutter heute noch, wenn sie irgendwo schlechten Kaffee getrunken hat».

14. - Vgl. z.B. «Einnerung mit Bäumen», S. 70: «Form, Geräusch und Geruch bilden eine Einheit, die nicht mehr in ihre einzelnen Komponenten zerlegbar ist», oder «Erinnerung mit Bäumen», S. 81: «Die Erinnerung überspringt die Jahrzehnte, als wären sie nicht gewesen».

15. - «Erinnerung mit Bäumen», S. 46.

16. - Vgl. z.B. «Erinnerung mit Bäumen» S. 118: «Vor dem Hof stehend, an jenem verregneten Junitag, habe sie sich mit der Sechzehnjährigen, die sie damals gewesen sei [...]. Nicht 
identifizeiren können» oder «Die Ahnenpyramide» S. 130: «... es kommt mir vor, als hätten die erinnerten Bilder nichts mit meiner eigenen Erfahrung zu tun...».

17. - S. 63: «Lassen sich Erinnerungen befehlen, liegt es an uns, welcher Teil eines Geschehens in unserem Gedächtnis bewahrt wird? Liegt es an der wahrscheinlich ständig zunehmenden Müdigkeit [...], daß von Willis weiterem Weg nicht so starke Eindrücke in seiner Erinnerung zurückgeblieben sind?».

18. - «Die Ahnenpyramide» S. 16.

19. - Ebenda S. 54.

20. - Es handelt sich um die Gegend von Schlierbach (Oberösterreich).

21. - Vgl. «Liebe alte Kinderreime» (S. 7): «Lirum Lamm Löffelstiel manche Kinder essen viel manche müssen fasten Napalm liegt im Kasten Bombe liegt daneben Ei welch ein lustig Leben !» oder die beiden «Abc» (S. 12 und 13): «Die Katze lief im Schnee. Und als sie wieder heraus kam da hatt sie weiße Stiefel an O Jemine o je!» - «Atomstaub ist im Schnee. Ein Kindchen ward geboren hat weder Mund noch Ohren O Jemine o je! O Jemine o je!».

22. - Vgl. «Zwischenbericht» im gleichnamigen Band, S. 5: «beim Schein der Kometen schreibe ich ruhig Worte und Worte auf weißes Papier», oder «Schleswig Holstein», auch in «Zwischenbericht» S. 61: «du stehst: (ein winziger Punkt) im Zentrum der Scheibe Welt».

\section{AUTEUR}

\section{CARINE KLEIBER}

Université de Haute Alsace 